\title{
COMPARATIVE STUDY ON ANTERIOR CRUCIATE LIGAMENT RECONSTRUCTION: DETERMINATION OF ISOMETRIC POINTS WITH AND WITHOUT NAVIGATION
}

\author{
Fabio J. Angelini, Roberto F. M. Albuquerque, Sandra U. Sasaki, Gilberto L. Camanho, Arnaldo J. Hernandez
}

doi: $10.1590 / \mathrm{S} 1807-59322010000700006$

Angelini FJ, Albuquerque RFM, Sasaki SU, Camanho GL, Hernandez AJ. Comparative study on anterior cruciate ligament reconstruction: determination of isometric points with and without navigation. Clinics. 2010;65(7):683-8.

OBJECTIVES: To compare the accuracy of tunnel placement and graft isometry for anterior cruciate ligament reconstruction performed using a computer-assisted navigation system (Orthopilot) and using traditional instruments.

METHODS: The anterior cruciate ligament was removed intact from 36 pairs of human cadaver knees. From each pair, one knee was randomized to Group 1 (conventional) and the other to Group 2 (Orthopilot). An inelastic suture was then passed through the central points of the tibial and femoral tunnels. Neither of the tunnels was drilled. All knees were then dissected, and six parameters were obtained: distances from the tibial tunnel center to the 1) posterior cruciate ligament, 2) anterior horn of the lateral meniscus and 3) medial tibial spine; 4) distance from the femoral tunnel center to the posterior femoral cortex; 5) femoral tunnel coronal angle; and 6) variation of the distance from the femoral to the tibial tunnel with the knee extended and at 90 degrees of flexion.

RESULTS: The variation of the distance from the femoral to the tibial tunnel during flexion and extension was smaller in the Orthopilot group (better isometry) compared to the conventional group. There were no statistical differences in any other parameters between the groups, and all tunnels were considered to be in satisfactory positions.

DISCUSSION: The results obtained for anterior cruciate ligament reconstruction depend on precise isometric point positioning, and a navigation system is a precision tool that can assist surgeons in tunnel positioning.

CONCLUSION: No differences in tunnel position were observed between the groups. Nonetheless, better isometry was achieved in the Orthopilot group than with conventional instruments.

KEYWORDS: Knee; Anterior cruciate ligament; Biomechanics; Navigation.

\section{INTRODUCTION}

Traumatic injury to the anterior cruciate ligament (ACL) of the knee presents with increasing frequency and is a common problem among the athletically active population. Patients who suffer this injury often develop clinical instability of the knee. Although conservative treatment has its indications, surgical treatment for ACL injuries is the ideal choice for young and active patients because it allows them to return to sports but prevents the onset of additional knee injuries. ${ }^{1}$

Instituto de Ortopedia e Traumatologia, Hospital das Clinicas, Faculdade de Medicina, Universidade de São Paulo - São Paulo/SP, Brasil.

Email: rmotaa@uol.com.br

Tel.: 5511 3214-4422

Received for publication on February 18, 2010

First review completed on March 03, 2010

Accepted for publication on April 14, 2010
Surgical techniques have evolved over recent decades, and the current gold standard treatment is arthroscopic intra-articular ACL reconstruction with autogenous grafts. The main aspects of the surgical technique required to achieve favorable clinical results include graft quality, tunnel positioning and efficient graft fixation, with sufficient tension to provide joint stability and a physiological range of motion with normal flexion and extension. ${ }^{2,3}$ Along with the increasing number of indications for surgery, increases in the frequency of revision surgery have also been reported. Revision surgery is now performed in $10-40 \%$ of cases. ${ }^{4}$ The main cause of surgical failure and ACL revision is inadequate tunnel positioning. ${ }^{5,6}$ Anatomical studies have reported that the anatomical ACL origin and insertion is the most isometric graft position ${ }^{7-10}$. However, it is known that even senior surgeons using conventional guides frequently fail to place the tunnels in the desired positions. ${ }^{11,12}$

A recent resource for improving orthopedic 
surgery precision is the Computer Assisted Orthopedic Surgery (CAOS) system. ${ }^{13}$ Navigation systems for ACL reconstruction have been developed, ${ }^{14-18}$ but no comparative clinical studies have unequivocally demonstrated their superiority in relation to conventional guides. In addition to enhancement of precision, such navigation systems can also facilitate choosing the location for bony tunnels based on the new isometry criterion. Before the advent of such systems, these data could not be supplied to surgeons intraoperatively. The purpose of the present study was to compare isometry and tunnel positioning using the CAOS system and using conventional guides.

\section{MATERIALS AND METHODS}

The inclusion criteria for the knee specimens used were that the cause of death was not traumatic or due to infectious disease and that there were no scars, hematomas or deformities in the lower limbs. The exclusion criteria diagnosed during arthroscopy were the presence of any ligament lesions, meniscal lesions or degenerative joint disease. None of the specimens examined was excluded.

\section{Specimen Preparation}

Thirty-six freshly frozen, undamaged human knees from cadavers (18 pairs from 18 cadavers) were used in this study. Four of the cadavers were female and 14 were male, ranging in age from 38 to 76 years (mean, 51.9; standard deviation (SD), 11.9). The femur was cut $20 \mathrm{~cm}$ and the tibia $30 \mathrm{~cm}$ from the joint line. The iliotibial tract up to mid-thigh, the popliteus musculotendinous unit and the joint capsule were left intact. The knees were stored at $-20{ }^{\circ} \mathrm{C}$ and thawed for 12 hours at room temperature before testing. Prior to the procedures, the knees were subjected to an initial arthroscopic inspection to rule out any previous intra-articular lesions.

\section{Groups}

The specimens were divided in two groups, each comprising one knee of each pair (randomized side distribution by means of flipping a coin). These groups were named Group 1, which consisted of 18 knees that underwent ACL reconstruction using conventional guides, and Group 2, which consisted of the 18 opposite-side knees that underwent ACL reconstruction assisted by the CAOS system and navigation instruments. All procedures were performed by the same surgeon, who had prior experience in ACL reconstruction and navigation.

\section{Surgical technique}

\section{Group 1: Conventional guide technique}

In Group 1, guide wire positioning was performed using conventional surgical instruments. For the tibial tunnel, an Acufex tibial guide was used (Director model; Smith \& Nephew, Inc., U.S.A.) with $55^{\circ}$ sagittal angulation and $15^{\circ}$ lateral inclination and with the tip of the guide directed toward the intercondylar eminence of the tibia, halfway between the anterior horn of the lateral meniscus and the medial tibial intercondylar tubercle. In this position, a fourhole wire guide was drilled from the anteromedial surface of the tibia to the tip of the guide inside the joint. The position of the guide wire was checked using an arthroscope. A polyester suture wire $\left(\right.$ Ethibond $^{\circledR} 5$ ) was then pulled by the metal guide wire from the extra-articular anteromedial cortical surface of the tibia to the inside of the joint, exiting from the central point of the tibial tunnel, which was not drilled (Figure 1).

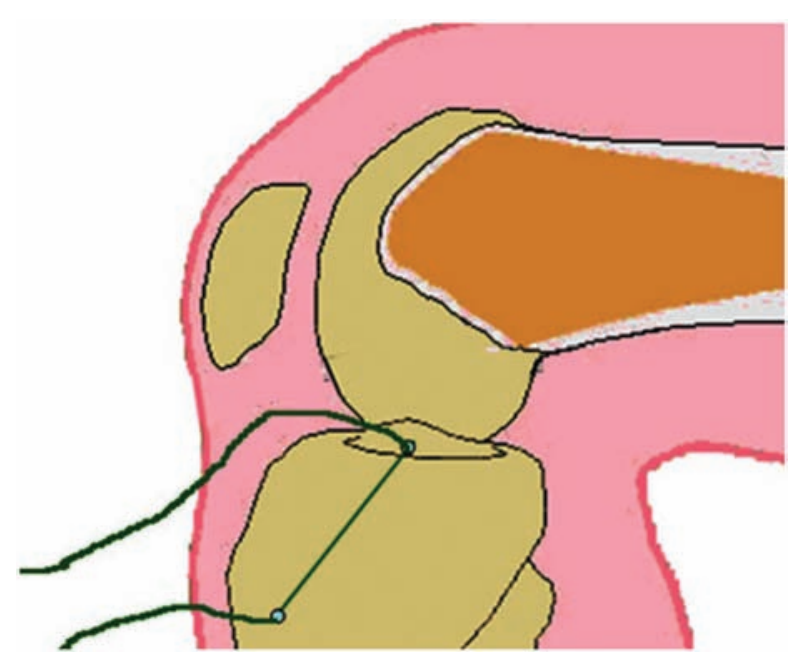

Figure 1 - The suture wire was pulled through the tibia into the knee after drilling with the guide wire.

For the femoral tunnel, a 7-mm offset Acufex femoral guide was introduced through the anteromedial portal and positioned at the femoral intercondylar notch, with the tip of the guide on the posterior wall at eleven o'clock for the right knee and one o'clock for the left knee. In this position, the four-hole wire guide was drilled from the medial surface of the lateral femoral condyle to the anterolateral surface of the thigh of the specimen. The end of the suture wire that had been left inside the joint was then pulled through the anteromedial portal and was led by the four-hole wire guide through the femur (Figure 2). In this way, the suture wire was positioned at the central point of the femoral tunnel, which was also not drilled. 


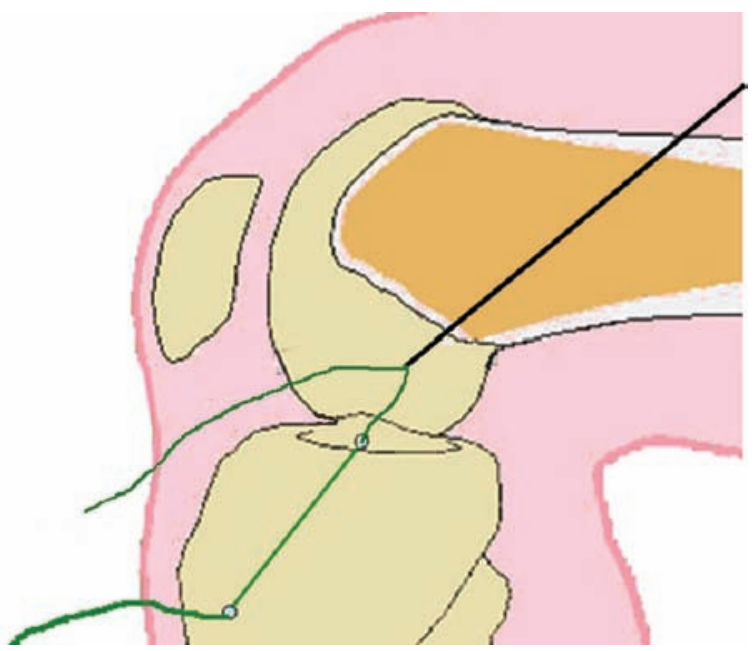

Figure 2 - The suture wire was pulled through the femur by the guide wire out to the anteromedial femoral metaphyseal surface.

\section{Group 2 - Navigation technique}

Group 2 guide wires were positioned with assistance from the Orthopilot navigation system. Orthopilot ${ }^{R}$ (Aesculap, Tuttlingen, Germany) is a computer system that provides three-dimensional (3D) real-time tracking of specific surgical instruments in relation to anatomical reference points that are acquired during surgery. The 3D tracking is performed without the need for additional preoperative planning or imaging such as tomography. The anterior cruciate ligament replacement software, version 1.1 (2002) was used together with its specific surgical instruments. To provide tracking, two passive rigid bodies with four reflective spheres each were attached using K-wires to the femur and tibia. Infrared rays were provided by a source at the side of the cameras. The hardwired cameras detected the signals reflected by the spheres and determined their spatial position. A third passive rigid body was connected to the specific instruments for each step of the surgery.

In the Orthopilot setup, the graft diameter was set to $10 \mathrm{~mm}$ for all knees. The anatomical reference points and kinematics of the knee were entered and registered by the Orthopilot software, thus allowing it to calculate the relative positions between the fixed sensors and anatomical landmarks.

The anatomical reference points input to the system were obtained using a pointer connected to the mobile and passive rigid bodies (Figure 3). Superficial anatomical landmarks were registered in sequence, starting with the anterior tibial tuberosity and extending to the anterior, medial and lateral tibial plateau borders. The following intra-articular structures were viewed by means of arthroscopy and registered with the pointer through the anteromedial portal: posterior cruciate ligament (PCL), anterior tibial insertion, medial intercondylar tubercle, posteromedial margin of the

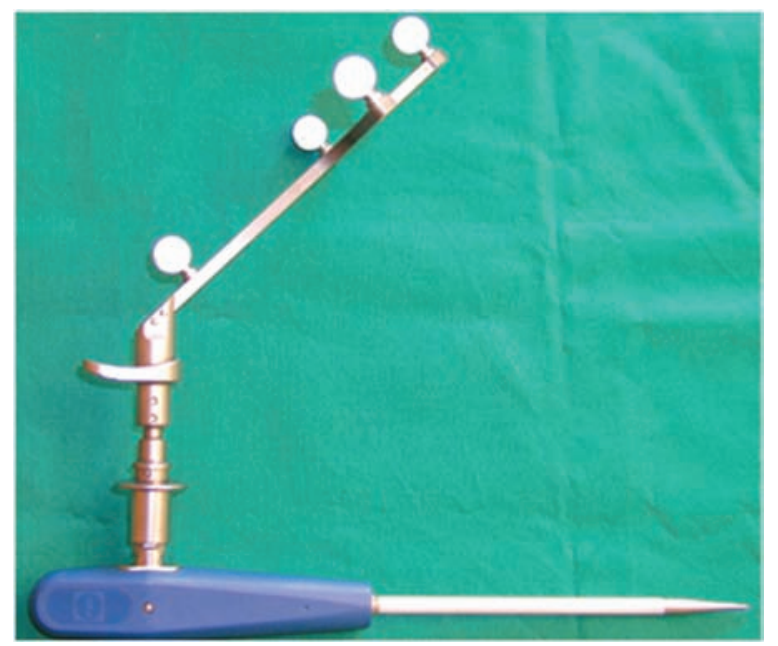

Figure 3 - Rigid body with reflective spheres for the navigation-linked pointer.

anterior horn of the lateral meniscus, at least five points of the anterior border of the femoral intercondylar notch at its transition with the joint cartilage and at least five points on the medial surface of the femoral lateral condyle.

Subsequent palpation of the posterior wall of the femoral intercondylar notch in two positions, eleven and twelve o'clock for the right knee and twelve and one o'clock for the left knee, was carried out with a navigation-linked pointer.

The first step in the navigation was the positioning of the tibial tunnel; a specific tibial guide connected to the mobile and passive rigid bodies was tracked, and its position was monitored on the computer screen (Figure 4). The target parameters used were the same as those described for Group 1. As soon as the desired position of the tibial guide was achieved, the guide wire was drilled through the guide from the tibial anteromedial surface to the guide tip inside the joint. A polyester suture wire $\left(\right.$ Ethibond $^{\mathrm{R}} 5$ ) was pulled through the four-hole wire guide along the drilled path. The wire remained free inside the joint at the center of what would be the tibial tunnel, which was not drilled as in Group 1.

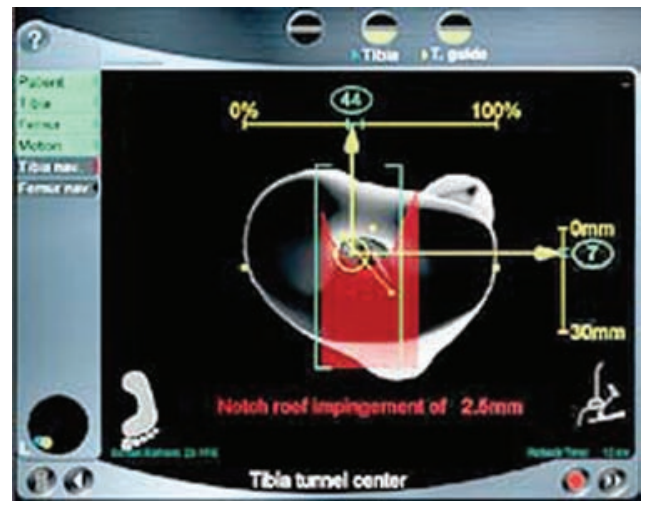

Figure 4 - Tibial guide navigation screen. Target parameters correspond to the green interval on the superior and lateral scales. 
The data for the tibial tunnel were stored in the computer and used to calculate the optimum entry point for the femoral tunnel in relation to that specific tibial tunnel.

A navigated femoral guide was introduced through the anteromedial portal and was tracked by the navigation system, allowing the guide position on the medial wall of the lateral femoral condyle to be followed on the computer screen. In addition, it permitted the calculation of the following parameters: coronal angulation, distance from the femur posterior wall and graft isometry.

The point selected for the center of the femoral tunnel respected the previously defined parameters and provided the best isometry. In this position, the four-hole wire guide was drilled from the medial surface of the lateral femur condyle to the anterolateral surface of the thigh of the specimen. The same procedures as followed for Group 1 were performed in relation to the suture wire, and the femoral tunnel was not drilled. Thus, no tunnel was drilled, but the suture wire was positioned inside the knees, through the centers of what would be the femoral and tibial tunnels.

The isometry measurement methods were based on those described by Hernandez et al. (1995). A knot was made at the end of the suture wire, proximally to the femur. This knot had a diameter larger than $2.5 \mathrm{~mm}$, and therefore, when the opposite end emerging from the tibia was pulled, it could not migrate beyond the femoral cortex. Another knot was made at the extra-articular tibial end of the wire at an arbitrary distance from its tibial exit. The wire length between the tibial exit and the distal knot, with the knee at $90^{\circ}$ of flexion and total extension (Figure 5), was measured using a manual pachymeter. The difference between these measurements corresponded to the difference between the distances from the femoral tunnel to the tibial tunnel at these positions. The graft was considered isometric if the length variation was zero.

Through arthrotomy, the distances to the PCL, medial intercondylar tubercle and margin of the anterior horn of the lateral meniscus relative to the guide wire exit on the tibial

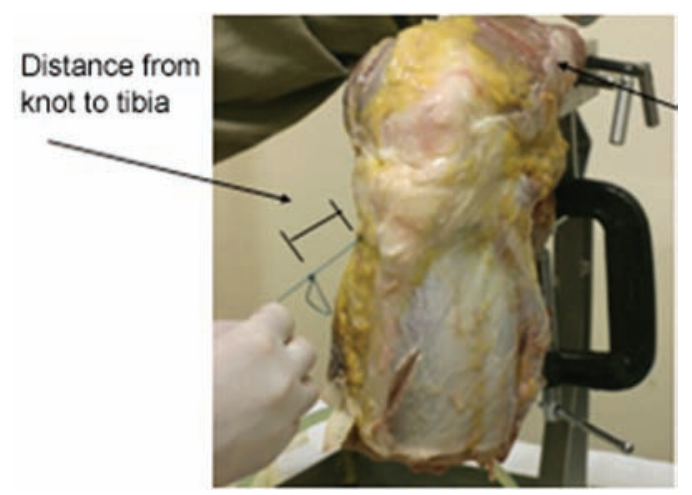

Figure 5 - Suture wire length variation. Difference in wire length between the tibial exit and the distal knot, with the knee at $90^{\circ}$ of flexion and at total extension. joint surface were collected using a manual pachymeter. The posterior wall thickness was measured on the femur.

A digital photograph of the frontal view of the femoral intercondylar notch was taken with $90^{\circ}$ knee flexion. A line perpendicular to the tibial joint surface was drawn through the center of the intercondylar notch (twelve o'clock). The intersection of this straight line with the image of the cranial edge of the tibia determined the point that was considered the angular vertex. From this point, a second straight line was drawn through the center of the femoral tunnel. The angle formed by these two straight lines was measured by the software (Figure 6).

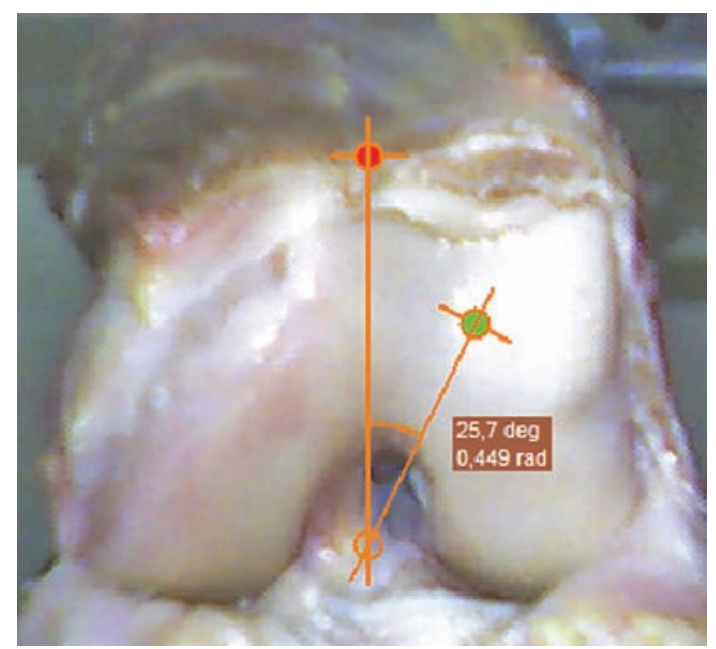

Figure 6 - Femoral tunnel angular position in the notch. The angle was measured with software.

\section{Statistics}

The data were analyzed using the Wilcoxon test for comparisons between two paired samples. A significance level of 0.05 was used $(\mathrm{p} \leq 0.05)$.

\section{RESULTS}

The variation of the distance from the femoral tunnel to the tibial tunnel between flexion and extension was smaller in the Orthopilot group [better isometry; Group 1, mean $=4.2 \mathrm{~mm}$ (range, 1.1-9.4 mm); Group 2, mean $=2.8 \mathrm{~mm}$ (range, 0.3-6.7 mm); $<$ 0.05]. None of the other parameters showed any significant difference between the groups, and all of the tunnels were considered to be in satisfactory positions (Table 1).

\section{DISCUSSION}

The design of this study aimed to make simple comparisons using direct measurements between the 
Table 1 - Results

\begin{tabular}{|c|c|c|c|c|c|}
\hline & \multicolumn{2}{|c|}{ Group 1} & \multicolumn{2}{|c|}{ Group 2} & \multirow[t]{2}{*}{ Wilcoxon } \\
\hline & Mean & Standard deviation & Mean & Standard deviation & \\
\hline$\Delta \mathrm{TT}-\mathrm{LM}$ & 7.24 & 2.34 & 7.56 & 2.53 & $P=0.756$ \\
\hline$\Delta$ TT-MIT & 7.15 & 2.42 & 6.58 & 2.35 & $\mathrm{P}=0.459$ \\
\hline$\Delta$ TT-PCL & 10.24 & 3.58 & 10.16 & 3.58 & $P=0.931$ \\
\hline$\Delta$ FT-PW & 4.56 & 2.54 & 4.26 & 3.59 & $P=0.868$ \\
\hline$<\mathrm{FT}$ & 19.18 & 5.70 & 14.16 & 8.98 & $P=0.063$ \\
\hline$\neq \Delta$ FT-TT $0^{\circ}-90^{\circ}$ & 4.2 & 5.45 & 2.84 & 3.38 & $\mathrm{P}=0.028^{*}$ \\
\hline
\end{tabular}

$\Delta$ - distance; TT- tibial tunnel; LM- lateral meniscus; MIE- medial intercondylar tubercle; PCL- posterior cruciate ligament; FT- femoral tunnel; PW- posterior femoral wall; < FT- femoral tunnel angular position in the notch; $\neq$ - variation

positions of the ACL reconstruction tunnels achieved using the Orthopilot navigation system and using conventional guides. Furthermore, and perhaps more importantly, this study aimed to determine which of these techniques provided better isometry.

The knees used in this study did not present any ACL injury, and the ACLs were arthroscopically removed. The resection invariably leaves clear signs of the anatomical origin and insertion of the ACL, and this may have assisted in positioning the guides, thereby potentially improving the results in both groups.

Direct measurements using a manual pachymeter have the advantage of being simple and reproducible, particularly when performed by a single surgeon. The measurements were based on absolute points that were covered by radiolucent structures, including cartilage and other tissues. Thus, our data cannot be compared with radiographic data. ${ }^{19}$, ${ }^{20}$ The measurements of the distance from the tibial tunnel to the PCL showed averages close to $10.2 \mathrm{~mm}$ in both groups. This result was higher than the expected result of $7 \mathrm{~mm}$, potentially indicating that this was not a reliable reference point for tunnel positioning.

Measurement (in degrees) of the coronal angle of the femoral tunnel using digital photography has not been reported previously in the literature. We chose to use this measurement because of its reproducibility and because it is easy to perform.

Therefore, it was surprising that the two groups, which demonstrated no significant differences in any anatomical parameters, had different isometric behaviors. The Orthopilot group not only had a lower average but also a lower interval between the minimum and maximum values, and this result was very consistent.

Our interpretation of this finding is that there is a relatively broad acceptable area for positioning the centers of the tibial and femoral tunnels. Positioning the center of the tunnel at any of the infinite number of points inside these areas would be appropriate. Therefore, an infinite number of possible combinations of appropriate tibial tunnels with appropriate femoral tunnels for each knee are possible. Each of these combinations determines different mechanical graft behavior and different isometry.

The navigation system seemed to provide us with a femoral tunnel location (chosen after positioning the tibial tunnel) in an acceptable area of the femur in relation to the selected tibial position, thereby providing decreased variation of the graft length or better isometry. However, the navigation system presents some potential inconveniences, such as the longer time required for the procedure ${ }^{21}$ and morbidity related to the fixation of rigid bodies to bones. These factors were not evaluated in the present study.

The results of this study suggested that there was no difference in the anatomical tunnel position between the groups. However, further studies with a larger number of cases are needed to confirm these results. Finally, the results of our study encourage us to continue evaluating the use of navigation for the improvement of knee surgery.

\section{CONCLUSION}

There was no difference in anatomical tunnel position between the groups. Better isometry was achieved using the navigation technique (Group 2) compared to the conventional technique (Group 1).

\section{REFERENCES}

1. Kurzweil PR, Jackson DW. Chronic anterior cruciate ligament injuries. In Fu FH, Harner CD, Vince KG. Knee surgery. Baltimore: Williams \& Wilkins; 1994. p. 731-47.
2. Hoogland T, Hillen B. Intra-articular reconstruction of the anterior cruciate ligament. An experimental study of length changes in different ligament reconstructions. Clin Orthop Relat Res. 1984;185:197-202. 
3. Khalfayan EE, Sharkey PF, Alexander AH, Bruckner JD, Bynum EB. The relationship between tunnel placement and clinical results after anterior cruciate ligament reconstruction. Am J Sports Med. 1996;24:335-41.

4. Sati M, Staubli H, Bourquin Y, Kunz M, Nolte LP. Realtime computerized in situ guidance system for ACL graft placement. Comput Aided Surg. 2002; $7: 25-40$

5. Getelman MH, Friedman MJ. Revision anterior cruciate ligament reconstruction surgery. J Am Acad Orthop Surg. 1999;7:189-98.

6. Gali JC, Adad MAH, Mod MSB. Causas potenciais de recidiva da instabilidade após reconstrução do ligamento cruzado anterior. Rev Bras Ortop. 2005;40:52-9.

7. Odensten M, Gillquist J. Function anatomy of the anterior cruciate ligament and a rationale for reconstruction. J Bone Joint Surg Am. 1985,67:257-62.

8. Penner DA, Daniel DM, Wood P, Mishira D. An in vitro study of anterior cruciate ligament and isometry. Am J Sports Med. 1988;16:238-43.

9. Schutzer SF, Christen S, Jakob RP. Further observations on the isometricity of the anterior cruciate ligament. Clin Orthop Relat Res. $1989 ; 242: 247-55$

10. Hefzy MS, Grood ES, Noyes FR. Factors affecting the region of most isometric femoral attachments. Am J Sports Med. 1989;17: 208-16.

11. Eriksson E. How good are the results of ACL reconstruction? Knee Surg Sports Traumatol Arthrosc. 1997;5:137.

12. Sudhahar TA, Glasgow MMS, Donell ST. Comparison of expected vs. actual tunnel position in anterior cruciate ligament reconstruction. Knee. 2004;11:15-8.
13. Albuquerque RFM, Angelini FJ, Pécora JR, Amatuzzi MM, Sasaki SU. Computer-assisted knee total arthroplasty. Acta Ortop Bras. 2006;14:199-202.

14. Dessenne V, Lavallée S, Julliard R, Orti R, Martelli S, Cinquin P. Computer-assisted knee anterior cruciate ligament reconstruction: first clinical tests. J Image Guid Surg. 1995;1:59-64.

15. Julliard R, Lavallee $S$, Dessenne V. Computer assisted reconstruction of the anterior cruciate ligament. Clin Orthop Relat Res. 1998;354:57-64.

16. Sati M, Staubli H, Bourquin Y, Kunz M, Nolte LP. Realtime computerized in situ guidance system for ACL graft placement. Comput Aided Surg. $2002 ; 7: 25-40$

17. Müller-Alsbach UW, Staubli AE. Computer aided ACL reconstruction. Injury. 2004;35:S-A65-7.

18. Schep NWL, Stavenuiter HJ, Diekerhof CH, Martens EP, van Haeff CM, Broeders IA, et al. Intersurgeon variance in computer-assisted planning of anterior cruciate ligament reconstruction. Arthroscopy. 2005;21:9427.

19. Koh J, Koo SS, Leonard J, Kodali P. Anterior cruciate ligament (ACL) tunnel placement: a radiographic comparison between navigated versus manual ACL reconstruction. Orthopedics. 2006;29:S122-4.

20. Panisset JC, Boux De Casson F. Navigated anterior cruciate ligament reconstruction: correlation between computer data and radiographic measurements. Orthopedics. 2006;29:S133-6.

21. Koh J. Computer-assisted navigation and anterior cruciate ligament reconstruction: accuracy and outcomes. Orthopedics. 2005;28:S1283-7. 